\title{
Covid-19 Aftermath and Population Science's Research Agenda
}

Alberto Palloni

Follow this and additional works at: https://knowledgecommons.popcouncil.org/series_pdr_essays-covid How does access to this work benefit you? Let us know!

\section{Recommended Citation}

Palloni, Alberto. "Covid-19 Aftermath and Population Science's Research Agenda." In Covid-19 and the Global Demographic Research Agenda, edited by Landis MacKellar and Rachel Friedman, 47-53. New York: Population Council, 2021. 


\title{
Covid-19 Aftermath and Population Science's Research Agenda
}

\author{
Alberto Palloni
}

THERE ARE MANY DOMAINS where the Covid-19 pandemic will leave deep footprints on the world's populations. Albeit with unequal degree of detail, I consider three of these domains: infancy and early childhood, the elderly, and the "sandwich generation" of young adults. I assess the possible demographic impacts of the pandemic, identify key problems, and propose research questions and materials that may improve understanding of those impacts and contribute to designing interventions to attenuate the damage on future generations.

\section{Mothers, Children, Covid-19, and the Health and Well-being of Adults for the Rest of the Twenty-First Century}

Assessments of the potential long-term impact of Covid-19 on individuals who will become older adults 50 to 60 years from now should consider several types of exposures: parents about to conceive, embryos soon after zygote formation and implantation, fetuses during uterine life, infants breastfed during the pandemic, and young children whose physical and cognitive development is entirely dependent on parental resources, preferences, and behaviors. The delayed manifestation of exposure to Covid-19 will be a function of when during these windows of time the exposure took place, its intensity, and its duration.

We know that, as a consequence of multiple interventions designed to arrest transmission of the virus, virtually all affected populations experienced at some point irregularities in food supply, sharp rises in unemployment, decreases in household income, increased isolation and multiplication of stressful conditions, and weakened access to health care. We also know that these conditions were not shared equally, either within borders or internationally: 
poor, marginalized, vulnerable populations are everywhere disproportionately affected, and these account for a higher share of population in low- and middle-income countries than in high-income countries.

There are three main pathways from exposures to Covid-19 pandemic conditions and delayed effects on offspring. The first is via nutritional constraints, the second is a function of parental stress, and the third depends on the direct impact of the virus on pregnant mothers or mothers who deliver during the pandemic. As to the first, despite short-term policy interventions, the decline in household income induced by prolonged unemployment and disruption of livelihoods will become a lingering feature of the pandemic, particularly among poor and marginalized populations in all countries. Deficiencies in paternal and maternal nutritional status (poor caloric intake and a nutrient-deficient diet) right before and during pregnancy impair fetal growth and derail early development via a combination of responses, some with immediate and some with delayed effects. Poor maternal health status can perturb the normal course of a pregnancy and complicate delivery, and maternal viral infections during pregnancy may compromise fetal development. Quality and length of breastfeeding depend on maternal nutrition and maternal stress. Also, unlike in-utero growth, mother-infant feeding is not mediated by barriers, so poor maternal diet directly exposes the child to risks that may have immediate and long-term effects. Empirical evidence from animal studies suggests that maternal depression and stress during some stages of pregnancy promote changes similar to those associated with deficient maternal diets. Further, it is known that postnatal parental stress leads to offspring depression and a heightened stress response, which has been associated with hypertension, cardiovascular disease, obesity, and metabolic disorders.

These considerations suggest that, all else constant, the long-term effect of the pandemic may be an increase in cohort-specific cognitive and chronic conditions due to exposure in utero and during infancy. Because the impacts of Covid-19 are unequally distributed within a population, these delayed effects will reproduce or augment current adult health and mortality inequalities. Individuals with limited resources who are less able to cope under normal conditions are far more likely to contract Covid-19 and less likely to experience a smooth recovery. Since early-infant and childhood health also influences educational attainment and adult labor-market success, the health-related effects of the pandemic can also eventually exacerbate socioeconomic inequality. To this we must add other inequality-enhancing mechanisms triggered by responses to the pandemic, such as those associated with the quality of learning-schooling disparities that result from adoption of online instruction, disruption and discontinuation of school attendance, or family displacement. 


\section{The Contribution of Population Research}

Population researchers could contribute to tackling these problems in two ways. First, based on an existing rich knowledge base, they could formulate new studies to shed light on long-term consequences, using as a foundation the investigation of problems that have been identified by studies of the 1918 pandemic. Fifty or 100 years from now, population sciences will want answers to questions such as the following. To what extent did children who were in utero or infants during the Covid-19 pandemic experience limitations in physical growth, excess illnesses, metabolic dysregulation, obesity, allergies, cognitive deficiencies? How do these children compare to those who were directly exposed to maternal infection in utero or contracted the virus in infancy and during early childhood? What are the social and economic gradients in these outcomes? Did these children experience different health conditions when they became adolescents and adults? Did they follow different educational and occupational trajectories and have differential labor market success? To what extent did their experience contribute to health and socioeconomic inequalities over their life course that will be manifested 50 or 100 years from now?

There are a number of ways to position population sciences to investigate such questions. A first option is to initiate new mother-child cohort studies that combine data collection on experiences with Covid-19, family exposures, and coping strategies with retrieval of biomarkers at regular intervals of time. Draw a national sample of females who are pregnant during the pandemic, those who give birth and their children aged 0-4. Gather hospital records with information about pre-pregnancy, pregnancy, and delivery; retrieve biomarkers from mothers and children; and establish a protocol to follow them for a period of time until, say, physical growth comes to a halt. This will result in a body of data ranging from conventional demographic and socioeconomic information to markers of physical growth, cognitive scores, school performance, and exposure to risk behaviors (poor diet, lack of exercise, smoking). With suitable identifiers and consent, the data-collection effort will make possible the formulation of additional studies to investigate outcomes at different stages of the life cycle of the same population. Some countries (Spain) are already planning data collection of this sort.

A second possibility is to piggyback on existing studies such as, in the United States, the National Health and Nutrition Examination Survey (NHANES), the Adolescent Health Study (AddHealth), the Fragile Families Study, or even the Early Childhood Longitudinal Study (ECLS) survey series. While not all include mothers and their children as targets, it is possible to either add new subsamples of mother-children pairs or, alternatively, recruit children of current participants. While most of the data needed are retrievable via traditional interviews and biomarker collection, an important enhance- 
ment would be to link mother-children pairs to health and hospital records in order to add clinical diagnosis and evidence of treatment. As these studies are likely to remain in place and already include representation of minorities and disadvantaged groups, they could become vehicles for collecting cohortlike information that comes close to, but is less expensive than, new cohort studies built from scratch.

A third possibility consists of selecting small urban and rural areas within larger regions, obtaining access to hospital and health care centers, and identifying and sampling mother-child pairs. This has already been done in some European cities to study child obesity. This sample could then be paired with random samples from the same area and the entire population thus recruited followed for a number of years. If fielded in several areas (provinces, states), these region-based, quasi case-control studies should support inferences about heterogeneity of impacts.

\section{Elder Health, Covid-19, and the Intergenerational Social Contract}

Although there are still many unknowns, it is clear that the near- and medium-term health effects of Covid-19 on the elderly will dwarf those experienced in younger populations, for a number of reasons, including high prevalence of preexisting conditions. As a result, the brunt of mortality excess and Covid-19 sequelae will be experienced by the elderly. Two consequences of this pattern are relevant.

Covid-19-related mortality will change the frailty composition of elderly survivors and will induce a short-term improvement of healthy life expectancy. As a result, the average health-care burden on family and kin will contract somewhat, providing temporary respite to younger cohorts and especially women. However, because of lingering aftereffects among elderly Covid-19 survivors, it is quite possible that these short-term impacts will reverse course when these individuals attain older ages. As a result, the brief increase in healthy life expectancy will be followed by a drop and the decrease in expected years in disability will reverse into an increase. The resulting expansion of demand for health care will compound existing pressure on the health-care system. To make matters worse, it will increase the burden of elder care in the younger generations, whose resources will be stretched as a consequence of Covid-19's impact on their own lives.

This situation has implications for the intergenerational social contract. Most societies have in place systems sustaining intergenerational monetary, in-kind, and time transfers that still closely involve family and kin. In some cases, the direction of transfers is from children to elderly parents, while in others it is the reverse. The direction, quantity, and nature of flows depends on traditional norms, social class, aggregate economic conditions, and the 
state of the nonfamilial intergenerational transfer system. It is likely that the effects of the pandemic will add stress to these family- and kin-based intergenerational flows for reasons set forth above. As a result, there will be increased pressure to develop or expand existing nonfamily and nonkin-based forms of transfers, essentially public. All this will be at a time when governments will be under post-crisis fiscal pressure, strapped by debt they were obliged to take on to sustain the near-term emergency response.

\section{The Contribution of Population Research}

Population science is exceptionally well positioned to meet the research challenge posed by these problems. There are now in place many longitudinal surveys of the elderly: the Health and Retirement Survey (HRS) in the US, the collection of Surveys of Health, Ageing and Retirement in Europe (SHARE) in many European countries, the English Longitudinal Study of Ageing (ELSA) in the UK, and many others. To turn these into tools for collecting information needed to study both the swings of healthy life expectancy and the strained intergenerational social contract is neither difficult nor expensive. In contrast to the case of research on children, the key challenge will be to create a study design guided by the right questions, not the collection of more information. Piggybacking on these surveys is feasible without refreshing samples beyond what these studies already plan to do. It only requires the addition of new questions in modules of intergenerational transfers, self-reported conditions, activities of daily living (ADL) and instrumental activities of daily living (IADL), and intergenerational transfers and assistance.

Two new protocols could be added to those already in existence. First is the collection of health records of survey participants who survived the infection and those who died as a consequence of it. These data will provide rich information related to diagnostic and treatment. Second, in those cases where DNA collection has already been done, an effort should be made to draw new biomarkers using saliva, full or dried blood spot samples. Among other problems, this will support investigations aimed at identifying genomic regions associated with severity of symptoms (e.g, the ACE2 gene).

\section{Young Adults, Covid-19, Closure of Opportunities and the Diffusion of Hopelessness}

What about the "sandwich generation" - those who when the pandemic struck were in high school, college, or had recently married and initiated careers, or are forced to drop out of the labor force to care for young children? Due to a number of reasons, adolescents and young adults have so far experienced the bulk of infections. However, the short-term course of the disease appears to be quite mild in this population. It is possible that, even if 
reinfection occurs, its effects will not be more serious than those experienced during the first episode.

While these considerations suggest a rather benign outlook, there are two consequences of the pandemic that could leave deep scars in these cohorts. First, there is some empirical evidence that the virus causes organ damage in a subset of otherwise healthy individuals, even among those who experience only mild effects. Symptoms include loss of memory, cognitive impairment, sleep disruption, and a number of physiological anomalies such as respiratory insufficiency, kidney dysfunction, and heart irregularities. Although not much is known about the risk factors associated with these responses or about their duration, there is growing concern that they may compromise the affected individuals' capacity to resume normal activities at work, in school, and in the household. Furthermore, we will not know for some time whether, in addition to these short-term responses, there will be others that will be felt as delayed effects in later adult life.

Second, members of these cohorts are most likely to experience the brunt of all the long-term nonhealth-related impacts of the pandemic. A list of these is long and may include a number of economic outcomes ranging from failure to get a good career start, to unstable incomes due to precarious employment and frequent spells of unemployment, resulting in an inability to save. All this with a powerful gender bias and amplifying existing inequalities between those who will be able to resume their planned economic life course after normality is reestablished and those who will find themselves irretrievably displaced from it.

These economic impacts will have consequences of their own, some of which may be union postponement and disruption, fertility limitation, delayed or foregone home purchase, and lack of resources to defray children's education costs. Some of these are consequential for the young adults' offspring, who will grow up in deprived environments and will see their own future compromised, as well. Finally, in the US, at least, these potential effects may combine to worsen current conditions that produce young adult hopelessness and poor future outlooks all of which result in the promotion of unhealthy behaviors such as smoking, alcohol and drug consumption/addiction, and other self-destructive habits that translate into excess adult mortality.

\section{The Contribution of Population Research}

The question that the grandchildren of today's population researchers might want to investigate is not just how the pandemic wreaked havoc on today's cohorts of young adults, but how, through the damage it inflicted on the parental generation, it transmitted damage to the next generation. Many countries have in place studies that could be modified to take on the task of collecting suitable information and investigating these issues. For example, in 
the US, there are surveys such as the National Longitudinal Survey of Youth (NLSY), the Survey of Income Program Participation (SIPPS), the Panel Survey of Income Dynamics (PSID), and perhaps even the Current Population Survey (CPS), all data-collection enterprises well equipped for the task. There is probably not even a need to recruit new participants (aside from those already planned). Much as in the case of elderly surveys, it suffices to tinker with the standard questionnaires by adding items or entire modules. Furthermore, in most developed and some developing countries, governments have administrative data on employment, wages, income, education, household composition, and, sometimes, health/mortality. These data can also often be linked to census-type surveys to fill in gaps in the purely administrative data and complement the information retrieved from longitudinal studies.

\section{Summary}

Admittedly, the study of the long-term effects of exposure among infants and children is a tall order. We are, however, in possession of knowledge and techniques that did not exist in 1918 and, unlike scientists then, we understand quite well the potential long reach of a shock of the magnitude and duration of Covid-19. The marginal costs of piggybacking on existing studies are in all likelihood an order of magnitude lower than those associated with a new cohort study. At the very least we should pursue one or a series of these and be always guided by what we have learned in the debate about the 1918 pandemic. Irrespective of the type of study we put in place, an overarching issue should be the intergenerational transmission of damage. The assessment of impacts on the elderly is a less taxing enterprise but it too requires a focus on problems that affect both the older generations and, simultaneously, their children and grandchildren. Investigation of these issues will be considerably enhanced by the fact that the instruments of today's population scientists are far superior to those available to researchers who lived through the $1918 \mathrm{flu}$. We have highly refined machinery to collect, link, organize, and process empirical data. We are in possession of more sophisticated analytical techniques to find patterns in the data and test conjectures. And, not insignificantly, we have vastly superior knowledge of the mechanisms that can produce longterm effects after exogenous shocks of similar scale. 